\section{Goodall and Gombe}

\author{
W.C. McGrew
}

The Chimpanzees of Gombe: Patterns of Behavior. By Jane Goodall.

Harvard University Press: 1986. Pp. 673. \$30, £19.95.

JANE Goodall is simply the most famous primatologist ever. Even if someone has never read her Animal Behaviour Monograph (1968), reporting her scientific studies of chimpanzees, or her more popular books, My Friends the Wild Chimpanzees (1967) and In the Shadow of Man (1971), she or he has probably seen one of Dr Goodall's wildlife documentary films. This alone means that any book of hers would deserve attention, for in a certain sense she defines the science for the world at large, in much the same way that Margaret Mead did for cultural anthropology.

In this case, the book will generate even more excitement, for it is the long-awaited updating of the Gombe story. The author produced her three previous books in little over ten years after starting in 1960 her pioneering field study of the long-haired Pan troglodytes of western Tanzania. But in the past 15 years there has been nothing, at least in book form. So, to which of her earlier books is this a sequel? Perhaps surprisingly, it tries to be all three. Like $M y$ Friends it is lavishly illustrated, with over 350 photographs. Like the monograph, it is full of tables, graphs and references. Like Shadow, it is a rich description of the lives and times of a community of individuals. The ambitiousness of the attempt is the source of both its strengths and weaknesses.

There are 20 chapters in the book, plus five appendices. Thirteen of the 20 are "data chapters", in that they report the analyses of quantitative data. The topics covered are what one would hope to find: demography, dominance, hunting, sex, territoriality, object manipulation and so on. These bring up to date the findings of recent years, and in some cases drive home a point which Dr Goodall has stressed repeatedly: that had study of Gombe's apes stopped after ten years (which is after all already a very long study by usual standards), our conclusions would have been incomplete and even misleading. The most graphic example is that of the extermination of the Kahama community by the Kasakela community, in a ruthless, one-by-one fashion. There was no hint of this until 1974, and till then systematic pongicide was undreamed of. Other results serve to confirm earlier suspicions, for example that female chimpanzees may also hunt, kill and eat mammalian prey, even fast-moving arboreal monkeys, under certain circumstances. Sex differences in predation persist, but simplistic generalizations (by this reviewer as well as others) about which sex does what are shown to be just that. Finally, other results are remarkable by their consistency: little has emerged about diet or the use of tools which was not known in the first 10 15 years. Instead, what is impressive is the conservatism of the material culture of

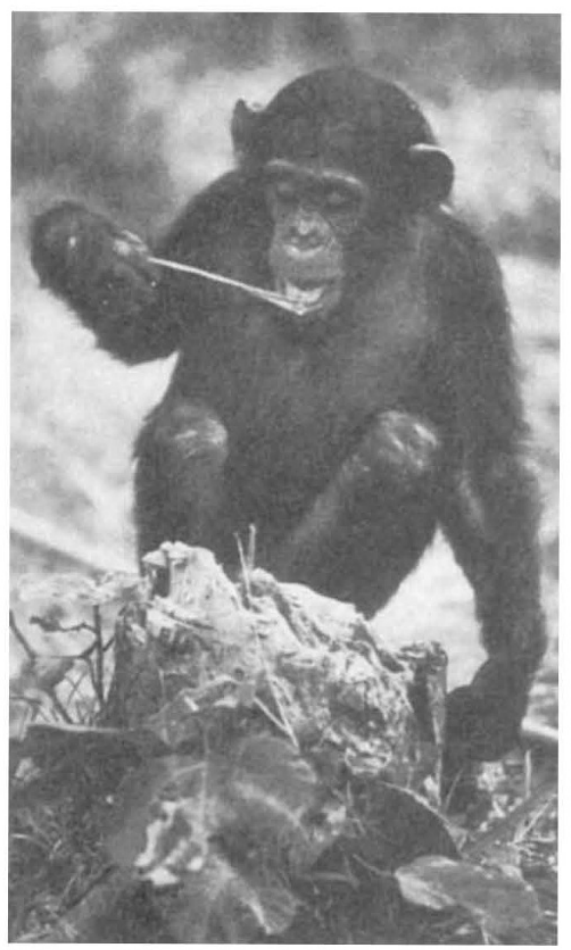

Tool use - Flint, son of Flo, fishing for termites with a blade of grass. (H. van Lawick; reproduced from The Chimpanzees of Gombe.)

wild chimpanzees, in that no innovation has been seen in 25 years.

The wealth of data is sometimes almost staggering: averages of up to 638 hours of observation per subject per year; 59 individuals followed from birth; 374 kills of vertebrate prey. Sadly, however, the analyses do not do justice to the data-set. Much hard-won information remains unreported, unanalysed and perhaps even untabulated because of Dr Goodall's resistance to using a computer. When data are presented, they are often difficult to interpret, as means (but not variances) and frequencies (but not rates) are often all that is given. Data are usually pooled across subjects, as for example when looking at the age and sex differences, so that independence of observations is compromised. Statistical tests are few - the first does not appear until p. 259 - and largely unexplained and mostly wrongly applied. Arguably, this might not matter in a semi-popular account, but two main points persist: one is that an immense amount of work has gone into datacrunching, and it is a pity to see this illserved. More seriously, it is usually not possible to tell whether apparently key results are real or not. For example, the graphs comparing male and female feeding times are said to be similar; in fact they are statistically significantly different. Or, it is said that distances travelled are much shorter on the day after a long excursion, but the tabulated data show such sequences to be random. One wonders if the book was ever scientifically edited.

The non-quantitative chapters are of mixed quality. The one on laboratory studies of chimpanzees skims selectively over well-known ground, focusing mostly on older work. Early "pongo-linguistics" is accepted uncritically, but (for example) Savage-Rumbaugh's elegant recent work on symbolic abilities is virtually ignored. The chapter might better have been omitted altogether, as it is of doubtful relevance. On the other hand, the chapter on "who's who" is masterly. In 18 pages, Dr Goodall presents pen-portraits of 29 chimpanzees with imagery that serves to keep the characters vividly alive throughout the book. A keen eye for individual differences has always been one of the author's strengths, and this is nowhere better shown than in the chapter on social awareness. She uses the apt anecdote at its sharpest to tackle convincingly the trickiest questions of social manipulation such as intentional deception.

All in all, the result is monumental, in several senses of the word. First, the book is massive: almost 700 pages, in large format with a smallish typeface, so that the text alone exceeds 350,000 words. Despite this it is priced so reasonably that even undergraduates will be able to afford it. Secondly, it chronicles 25 years of continuous research on our closest living relations. It is both comprehensive and comprehensible (she writes as clearly as ever), and it is the best such effort since Schaller's The Mountain Gorilla. Finally, it is a celebration of a cast of unforgettable characters, some now dead like old Flo, but others now matured, like Goblin, an infant when the study began but now the dominant male. Few people (Stella Brewer and Frans de Waal are two others who come to mind) can really enter the mind of the chimpanzee and then emerge to write about it well, but Jane Goodall is indisputably the best.

W. C. McGrew, a Senior Lecturer in the Department of Psychology, University of Stirling, Stirling FK9 $4 L A$, UK, is currently a visiting faculty member in the Departments of Anthropology and Biology, University of New Mexico, Albuquerque, USA. 